\title{
Vocal Tract Discomfort Scale (VTDS) and Voice Symptom Scale (VoiSS) in the Early Identification of Italian Teachers with Voice Disorders
}

\author{
Bruno Galletti ${ }^{1}$ Federico Sireci ${ }^{1,2}$ Roberta Mollica ${ }^{1}$ Elisabetta lacona ${ }^{3}$ Francesco Freni ${ }^{1}$ \\ Francesco Martines ${ }^{4}$ Enrique Perellò Scherdel ${ }^{5}$ Rocco Bruno ${ }^{1}$ Patrizia Longo ${ }^{1}$ Francesco Galletti ${ }^{1}$
}

\footnotetext{
${ }^{1}$ Department of Adult and Development Age Human Pathology "Gaetano Barresi," unit of otorhinolaryngology, University of Messina, Messina, Italy

2 Section of ENT, ARNAS Fatebenefratelli Hospital, Palermo Italy

${ }^{3}$ Section of Audiology, Fondazione IRCCS Ca'Granda Ospedale

Maggiore Policlinico, Milan, Italy

${ }^{4}$ Department Bio.Ne.C., Section of ENT, University of Palermo,

Palermo Italy

${ }^{5}$ Servicio de Otorrinolaringologia, Hospital General Universitari vall

D’Hebron Universitat Autonoma de Barcelona, Catalunya, Spain
}

Address for correspondence Federico Sireci, MD, Department of Adult and Development Age Human Pathology "Gaetano Barresi," unit of Otorhinolaryngology, University of Messina, Via Consolare Valeria 1, 98125, Messina ME, Italy (e-mail: federicosireci@hotmail.it).

Int Arch Otorhinolaryngol 2020;24(3):e323-e329.

\begin{abstract}
Keywords

- voice disorders

- VoiSS

- VTDS

- teachers

Introduction The current Italian law does not include any guidance regarding voice education, prevention of voice disorders and screening in subjects with high vocal loading such as teachers.

Objectives We aimed to check the correlation between the Vocal Tract Discomfort Scale (VTDS) with the Voice Symptom Scale (VoiSS) for the evaluation of Italian teachers. In addition, we aimed to investigate whether there are differences in the frequency and intensity of discomfort symptoms in teachers with disabilities comparing vocal tract discomfort symptoms in teachers with high risk (HRVD) and low risk (LRVD) of vocal disorders according to the VoiSS cutoff (>15.5).

Methods We analyzed 160 Italian teachers ( 111 women and 49 men) that completed the VTDS and VoiSS at vocal evaluation. The Spearman correlation test was applied to all variables. The Mann-Whitney $U$ test was used to compare the average number of discomfort symptoms among HRVD and LRVD teachers.

Results A moderate positive correlation was observed between the average number, frequency, and intensity of discomfort symptom and the total score, physical domain score, and limitation domain score of the VoiSS. Only the emotional domain score of the VoiSS showed a weak positive correlation $(p<0.001)$. Teachers considered in the HRVD group according to the VoiSS score had a higher number, frequency, and intensity of vocal tract discomfort symptoms.

Conclusion There is correlation between the VTDS and VoiSS scales. Like the VoiSS, the VTDS is a questionnaire that detects HRVD teachers. Therefore, the results suggest that both questionnaires could be useful for a preventive voice program for Italian teachers
\end{abstract}

received

February 15, 2019

accepted

September 17, 2019

published online

December 13, 2019
DOI https://doi.org/

10.1055/s-0039-1700586. ISSN 1809-9777.
Copyright $(2020$ by Thieme Revinter

Publicações Ltda, Rio de Janeiro, Brazil
License terms

()ㅇㅇㅛ 


\section{Introduction}

The voice represents an instrument for professionals that conduct activities involved in communication, information, art, culture, education and politics. To achieve effectively their communicative results, vocal professionals are exposed to vocal effort, making them susceptible to the development of vocal pathologies. ${ }^{1}$ Voice disorders in vocal professionals (occupational dysphonia) are part of dysfunctional dysphonia, caused by an incorrect production of the vocal signal (Malmenage) or an excessively prolonged use of the voice (Surmenage). ${ }^{2}$

Teachers showed the highest risk to develop voice disorders: they are subject to prolonged vocal effort, often in a work environment characterized by a high background noise and unfavorable acoustic conditions. ${ }^{3}$ Furthermore they may have inadequate postures, usually do not know the vocal hygiene standards and can deal with anxiety and stress situations. ${ }^{1}$

Studies reveal that $20 \%$ of teachers are subject to prolonged vocal effort. The prevalence of dysphonia is higher in teachers $(11 \%)$ than in other professional categories $(6.2 \%),{ }^{4}$ the prevalence for teachers at a single point in time ranged from 9 to $37 \%{ }^{5}$ the lifetime prevalence of voice disorders (teachers affected by dysphonia at some point in their lifetime) ranged from 50 to $80 \%$. $^{5,6}$

Vocal disorders can impact differently according to the profession: for vocal professionals, such as singers, actors, radio and TV hosts, teachers, lawyers, dysphonia has a higher effect on quality of life. In fact they are often the cause of social isolation and absence from work due to illness and patients underestimate the problem without wishing to undergo phonic examination with a fiber optic laryngoscope.

Through self-assessment tools, it is possible to know the sensations of the patient due to the vocal disorder, the awareness of dysphonia, and the impact of dysphonia on quality of life. ${ }^{7,8}$ The Vocal Tract Discomfort Scale (VTDS) is a self-assessed questionnaire for the subjective evaluation of voice disorders, proposed by Mathieson in 1993, commonly used in Great Britain. ${ }^{9}$ It measures the perception of discomfort in the vocal tract using eight qualitative descriptors according to the frequency and intensity of symptoms on a numerical scale from 0 to $6 .^{10}$ The subject attributes a score that best represents the frequency and intensity of each symptom: burning in the throat, throat tightness, dry throat, sore throat, itchy throat, sensitive throat, throat irritation and lump in the throat. Therefore, the questionnaire contains all of the symptoms that the vocal professionals underestimate and that are represented in cases of vocal disorders.

The Voice Symptom Scale (VoiSS) is a multidimensional questionnaire, divided into three scales (physical symptoms, vocal impairment, emotional impact), which investigates the frequency of occurrence of communication difficulties, vocal symptoms, stress and emotional impact of the voice disorder on the life of the patients ${ }^{1,7}$. The subject indicates the frequency with which he or she is affected by vocal symptoms. ${ }^{7}$ This questionnaire is already validated and used in Italy.
So far, in Italy, the VoiSS and VTDS are questionnaires diffused among the professional categories at risk for voice disorders, but there are few data in the literature especially for teachers. Amaral et al ${ }^{1}$ divided teachers according to the cutoff value of VoiSS into two groups: Vocally Healthy Group and Vocal Risk Group. The latter presented a worse VTDS score after the teaching activity. The VTDS is a short and rapid questionnaire, which could allow the early identification of voice disorder in teachers and could improve the compliance of teachers with preventive activities. For the first time in the literature, we aimed to check the correlation between the Vocal Tract Discomfort Scale (VTDS) with the Voice Symptom Scale (VoiSS), so as to demonstrate their combined use to improve the early detection of vocal disorders in Italian teachers without previous diagnosis. In addition, we aimed to investigate whether there are differences in the frequency and intensity of discomfort symptoms in teachers with disabilities comparing vocal tract discomfort symptoms in teachers with high risk (HRVD) and low risk (LRVD) of vocal disorders according to the VoiSS cutoff $(>15.5)$.

\section{Methods}

A retrospective, descriptive and cross-sectional study was performed in our Department of Otorhinolaryngology from 2017 to 2018 and was approved by the Ethics and Research Committee of our institution under the report number 1.202 .

After contacting school principals who promoted the possibility of participating in the project among dependent teachers, a total of 198 teachers have decided to join the initiative and signed an informed consent form about the realization of the study. These teachers were asked to complete the questionnaires at school before starting work to avoid bias related to stress and fatigue due to sustained lessons.

The inclusion criteria comprised teachers $>25$ and $<65$ years old, with a workload of 36 hours a week, with at least 5 years of service, and completion of the 2 protocols for selfassessment (VoiSS and VTDS).

The exclusion criteria were as follows: teachers who were on vacation, medical leave, or absent from teaching, who had previously undergone surgical or speech-language therapy, and who presented neurological, psychological and/or psychiatric alterations and who were diagnosed with vocal disorders on an organic basis by an otorhinolaryngologist; hormonal problems, use of medication, and smoking were disregarded ${ }^{11-14}$. These exclusion criteria were chosen to evaluate the effectiveness of the questionnaires in identifying teachers who do not have vocal diseases in place but are at professional risk for these disorders.

Considering these criteria, the study sample was composed of 160 Italian teachers, of which 111 (69.37\%) were women and 49 (30.62\%) were men, with a mean age of 50.05 ( \pm 8.26 standard deviation $[S D]$ ) years old and a mean 20.78 ( $\pm 9.57 \mathrm{SD}$ ) years of service. Of these teachers, 6 (3.75\%) were of nursery school, 28 (17.5\%) of primary school, 68 (42.5\%) of lower secondary school/junior high school, and 58 (36.25\%) of high school. Each teacher underwent a brief anamnesis (gender, age, kind of school, years of service) and completed 
the vocal self-assessment protocols (VoiSS and VTDS), both cross-culturally adapted into Italian. 8,15

The VTDS ${ }^{15-17}$ assesses the frequency and intensity of symptoms of discomfort of the vocal tract. It comprises eight qualitative patient-derived symptoms or sensations commonly referred to the vocal tract (burning, tight, dry, aching, tickling, sore, irritable, and lump in the throat). Each item is rated on a 7-point Likert scale (from $0-6$ ), both for its frequency (from "never" to "always") and its severity (from "none" to "extreme"). The total VTDS score ranges from $0-96$, whereas the score for each subscale ranges from $0-48$.

The following cutoff values were used, according to the literature, ${ }^{18}$ to indicate the presence or not of symptoms: $>0.5$ for burning, tightness, aching, tickling, and soreness, and $>2.5$ for dryness, irritation, and having a lump in the throat.

The Voiss ${ }^{19}$ investigated the frequency of occurrence of vocal symptoms. It includes 30 items, of which 15 are on the limitation domain (VOISS-L), 8 are on the emotional domain (VOISS-E), and 7 are on the physical domain (VOISS-P). Each item, according to the Likert scale, scored 5 points, numbered from 0 to 4 , with a total score ranging from $0-120$ (VOISS-T). The scores for the items in each domain were summed. The cutoff values used for the domains for this protocol were as follows: total $=15.5$, limitation domain $=11.5$, emotional domain $=1.53$, and physical domain $=6.5 .8,20$

For screening, the cutoff value of the Voice Symptom Scale (VoiSS $)^{8}$ was used to divide teachers into two groups: individuals with total VoiSS score $\geq 15.5$ points were allocated to the High Risk of Vocal Disorders (HRVD) group (102 teachers), whereas those with a total VoiSS score $<15.5$ points were allocated to the Low Risk of Vocal Disorders (LRVD) group (58 teachers). Both groups were compared to verify a possible relationship between I-VTDS and total I-VoiSS.

In addition, because we aimed to verify the relationships between each subscale of the two self-assessment instruments (VTDS and VoiSS), teachers were allocated in two subgroups: HRVD and LRVD according to the cutoff values of the VoiSS-T scale and of each subscale of the VoiSS. Therefore, we included in the group HRVD only those individuals who presented values above the cutoff point for the total score (VOISS-T) and for the specific subscale (VOISS-E, L, P). The LRVD group included teachers who presented values inferior to the cutoff point in the total score (VoiSS-T) as well as in the investigated subscale (VOISS-E, L, P).

\section{Data Analysis}

Statistical analyses were performed using IBM SPSS Statistics for Windows, Version 25.0 (IBM Corp., Armonk, NY, USA). The data are presented as means with SDs. The Spearman correlation test was applied to investigate the correlation between the VTDS and the VoiSS. The correlation coefficient presented a range from -1 to 1 and, according Bartz, ${ }^{21}$ we classified the grade of correlation in: very low (0.1-0.2), low (0.2-0.4), moderate $(0.4-0.6)$, strong $(0.6-0.8)$, and very high $(>0.8)$. The nonparametric Mann-Whitney U test was used to compare the average number, frequency, and intensity of the symptoms of discomfort between the HRVD and LRVD groups. The groups were created using the cutoff values of the different domains of the VoiSS (total, limitation domain, emotional domain, and physical domain). This test was designed to detect whether HRVD teachers would present a higher number, and greater frequency and intensity of vocal tract discomfort symptoms, as detected by the VTDS. A $p \leq 0.05$ was considered significant.

\section{Result}

Our study evidenced a statistically significant correlation $(p<0.05)$ between number, intensity and frequency of VTDS and the total and subscales of VoisS. The number of symptoms of discomfort showed a moderate correlation when compared with the total score, the limitation domain score, and the physical domain score $(p<0.001)$ of the VoiSS. Only the emotional domain score of the VoiSS showed a weak positive correlation $(p<0.001)$.

In particular it was a moderately positive correlation between the frequency and intensity of symptoms of discomfort of the vocal tract and the total score of the VoiSS overall about intensity and frequency of soreness. Similary, the physical score of the VoiSS evidenced a moderate positive correlation with VDTS, in particular for intensity of soreness ( $\mathrm{Cor}=0.61)$. Also, the limitation domain of VoiSS evidenced a moderate correlation with all symptoms of VTDS, but a weak correlation with aching intensity ( $\operatorname{cor}=0.37)$. A weak correlation was found between the frequency and intensity of symptoms of discomfort of the vocal tract and the emotional score of the VoiSS, except frequency of irritability and soreness and intensity of soreness ( $\mathbf{- T a b l e} \mathbf{1}$ ).

The VTDS differed significantly between teachers of the HRVD and of LRVD groups, with a greater frequency and intensity of symptoms in the first group. In particular, teachers considered as HRVD as detected by the number of symptoms, the VoiSS total ( - Table 2 ), limitation ( - Table 3 ), emotional ( - Table 4 ) and physical ( - Table 5 ) domain scores showed more symptoms of vocal tract discomfort than teachers in the LRVD group $(p<0.001)$.

\section{Discussion}

Alterations of the voice are often underestimated in the professional categories at risk. In particular, teachers exposed to vocal abuse present hoarseness, vocal fatigue and burning in the throat. Unfortunately, very often, despite these symptoms, patients underestimate the possibility of having a vocal disorder and do not undergo phoniatric examination. For this reason, the questionnaires can be a first tool to screen professional categories at risk and direct them toward a clinical and instrumental assessment. ${ }^{18}$

Therefore, qualitative and quantitative questionnaires of screening must be considered in the early identification of vocal disorders. Ours is the first study aimed to investigate the correlation between the scores of the VoiSs ${ }^{18}$ and the VTDS $^{6}$ in Italian teachers. Moreover, we investigated if 
Table 1 Correlation Between the Mean, Number, Frequency, and Intensity of Vocal Tract Discomfort Symptoms and the Voice Symptom Scale Scores

\begin{tabular}{|l|l|l|l|l|l|l|l|l|}
\hline & \multicolumn{2}{|l}{ VOISS-T } & \multicolumn{2}{l|}{ VOISS-L } & \multicolumn{2}{l|}{ VOISS-E } & \multicolumn{2}{l|}{ VOISS-P } \\
\hline Variables & Corr & $p$-value & Corr & $p$-value & Corr & $p$-value & Corr & $p$-value \\
\hline Number of VTDS & 0.52 & $<0.001$ & 0.45 & $<0.001$ & 0.30 & $<0.001$ & 0.62 & $<0.001$ \\
\hline Fr. Burning & 0.51 & $<0.001$ & 0.47 & $<0.001$ & 0.25 & $<0.001$ & 0.51 & $<0.001$ \\
\hline Fr. Tightness & 0.57 & $<0.001$ & 0.52 & $<0.001$ & 0.36 & $<0.001$ & 0.53 & $<0.001$ \\
\hline Fr. Dryness & 0.48 & $<0.001$ & 0.45 & $<0.001$ & 0.31 & $<0.001$ & 0.41 & $<0.001$ \\
\hline Fr. Aching & 0.53 & $<0.001$ & 0.42 & $<0.001$ & 0.40 & $<0.001$ & 0.57 & $<0.001$ \\
\hline Fr. Tickling & 0.48 & $<0.001$ & 0.42 & $<0.001$ & 0.31 & $<0.001$ & 0.47 & $<0.001$ \\
\hline Fr. Soreness & 0.61 & $<0.001$ & 0.53 & $<0.001$ & 0.46 & $<0.001$ & 0.57 & $<0.001$ \\
\hline Fr. irritability & 0.56 & $<0.001$ & 0.49 & $<0.001$ & 0.43 & $<0.001$ & 0.50 & $<0.001$ \\
\hline Fr. Iump in the throat & 0.52 & $<0.001$ & 0.43 & $<0.001$ & 0.39 & $<0.001$ & 0.53 & $<0.001$ \\
\hline Int. Burning & 0.48 & $<0.001$ & 0.42 & $<0.001$ & 0.31 & $<0.001$ & 0.52 & $<0.001$ \\
\hline Int. Tightness & 0.58 & $<0.001$ & 0.53 & $<0.001$ & 0.37 & $<0.001$ & 0.54 & $<0.001$ \\
\hline Int. Dryness & 0.51 & $<0.001$ & 0.46 & $<0.001$ & 0.31 & $<0.001$ & 0.43 & $<0.001$ \\
\hline Int. Aching & 0.49 & $<0.001$ & 0.37 & $<0.001$ & 0.38 & $<0.001$ & 0.59 & $<0.001$ \\
\hline Int. Tickling & 0.48 & $<0.001$ & 0.41 & $<0.001$ & 0.31 & $<0.001$ & 0.46 & $<0.001$ \\
\hline Int. Soreness & 0.63 & $<0.001$ & 0.54 & $<0.001$ & 0.48 & $<0.001$ & 0.61 & $<0.001$ \\
\hline Int. irritability & 0.53 & $<0.001$ & 0.47 & $<0.001$ & 0.38 & $<0.001$ & 0.48 & $<0.001$ \\
\hline Int. lump in the throat & 0.52 & $<0.001$ & 0.43 & $<0.001$ & 0.39 & $<0.001$ & 0.55 & $<0.001$ \\
\hline
\end{tabular}

Abbreviations: Corr., correlation; E, emotional; Fr., frequency; Int., intensity; L, limitation; P, physical; T, total; VoiSS, Voice Symptom Scale; VTDS, Vocal Tract Discomfort Scale.

Table 2 Comparison of the Mean, Number, Frequency, and Intensity of Vocal Tract Discomfort Symptoms in teachers with high risk (HRVD) and low risk (LRVD) of vocal disorders according to the Total Score of VoiSS

\begin{tabular}{|c|c|c|c|c|c|}
\hline \multirow[b]{3}{*}{ Variables } & \multicolumn{4}{|c|}{ VOISS-T } & \multirow[b]{3}{*}{ p-value } \\
\hline & \multicolumn{2}{|c|}{ HRVD } & \multicolumn{2}{|l|}{ LRVD } & \\
\hline & $\mathrm{M}$ & SD & $\mathrm{M}$ & SD & \\
\hline Number of VTDS & 2.33 & 1.15 & 1.20 & 1.03 & $<0.001$ \\
\hline Fr. burning & 2.92 & 1.30 & 1.77 & 1.49 & $<0.001$ \\
\hline Fr. tightness & 2.11 & 1.45 & 0.86 & 1.08 & $<0.001$ \\
\hline Fr. dryness & 2.90 & 1.33 & 1.39 & 1.34 & $<0.001$ \\
\hline Fr. aching & 2.35 & 1.28 & 1.20 & 1.07 & $<0.001$ \\
\hline Fr. tickling & 2.50 & 1.69 & 1.20 & 1.37 & $<0.001$ \\
\hline Fr. soreness & 2.93 & 1.51 & 1.25 & 1.29 & $<0.001$ \\
\hline Fr. irritability & 2.77 & 1.50 & 1.22 & 1.18 & $<0.001$ \\
\hline $\begin{array}{l}\text { Fr. lump in the } \\
\text { throat }\end{array}$ & 1.6 & 1.67 & 0.48 & 1.03 & $<0.001$ \\
\hline Int. Burning & 2.82 & 1.28 & 1.68 & 1.36 & $<0.001$ \\
\hline Int. tightness & 2.10 & 1.34 & 0.84 & 1.03 & $<0.001$ \\
\hline Int. dryness & 2.87 & 1.39 & 1.34 & 1.25 & $<0.001$ \\
\hline Int. aching & 2.48 & 1.36 & 1.34 & 1.20 & $<0.001$ \\
\hline Int. tickling & 2.5 & 1.75 & 1.17 & 1.41 & $<0.001$ \\
\hline Int. soreness & 2.71 & 1.39 & 1.10 & 1.05 & $<0.001$ \\
\hline Int. irritability & 2.06 & 1.49 & 1.20 & 1.13 & $<0.001$ \\
\hline $\begin{array}{l}\text { Int. lump in the } \\
\text { throat }\end{array}$ & 1.6 & 1.67 & 0.39 & 0.81 & $<0.001$ \\
\hline
\end{tabular}

Abbreviations: Fr., frequency; HRVD, high risk of vocal disorders; Int., intensity; LRVD, low risk of vocal disorders; SD, standard deviation; T, total; VoiSS, Voice Symptom Scale; VTDS, Vocal Tract Discomfort Scale. 
Table 3 Comparison of the Mean, Number, Frequency, and Intensity of Vocal Tract Discomfort Symptoms in teachers with high risk (HRVD) and low risk (LRVD) of vocal disorders according to the limitation Score of VoiSS

\begin{tabular}{|c|c|c|c|c|c|}
\hline \multirow[b]{3}{*}{ Variables } & \multicolumn{4}{|c|}{ VOISS-L } & \multirow[b]{3}{*}{ p-value } \\
\hline & \multicolumn{2}{|c|}{ HRVD } & \multicolumn{2}{|l|}{ LRVD } & \\
\hline & M & SD & M & SD & \\
\hline Number of VTDS & 2.83 & 1.29 & 1.35 & 1.05 & $<0.001$ \\
\hline Fr. burning & 3.10 & 1.33 & 1.77 & 1.49 & $<0.001$ \\
\hline Fr. tightness & 2.33 & 1.45 & 0.86 & 1.08 & $<0.001$ \\
\hline Fr. dryness & 2.88 & 1.29 & 1.39 & 1.34 & $<0.001$ \\
\hline Fr. aching & 2.44 & 1.33 & 1.20 & 1.07 & $<0.001$ \\
\hline Fr. tickling & 2.64 & 1.63 & 1.20 & 1.37 & $<0.001$ \\
\hline Fr. soreness & 2.79 & 1.44 & 1.25 & 1.29 & $<0.001$ \\
\hline Fr. irritability & 2.79 & 1.47 & 1.22 & 1.18 & $<0.001$ \\
\hline $\begin{array}{l}\text { Fr. lump in } \\
\text { the throat }\end{array}$ & 1.83 & 1.66 & 0.48 & 1.03 & $<0.001$ \\
\hline Int. Burning & 2.90 & 1.27 & 1.68 & 1.36 & $<0.001$ \\
\hline Int. tightness & 2.36 & 1.25 & 0.84 & 1.03 & $<0.001$ \\
\hline Int. dryness & 2.92 & 1.40 & 1.34 & 1.25 & $<0.001$ \\
\hline Int. aching & 2.53 & 1.42 & 1.34 & 1.20 & $<0.001$ \\
\hline Int. tickling & 2.65 & 1.69 & 1.17 & 1.41 & $<0.001$ \\
\hline Int. soreness & 2.80 & 1.44 & 1.10 & 1.05 & $<0.001$ \\
\hline Int. irritability & 2.67 & 1.49 & 1.20 & 1.13 & $<0.001$ \\
\hline $\begin{array}{l}\text { Int. lump in } \\
\text { the throat }\end{array}$ & 1.81 & 1.64 & 0.39 & 0.81 & $<0.001$ \\
\hline
\end{tabular}

Abbreviations: Fr., frequency; HRVD, high risk of vocal disorders; Int., intensity; L, limitation; LRVD, low risk of vocal disorders; SD, standard deviation; VoiSS, Voice Symptom Scale; VTDS, Vocal Tract Discomfort Scale.

those professionals considered at high risk of voice problems, as detected by the VoiSS, have a higher number and a greater frequency and intensity of vocal tract discomfort symptoms than teachers with a lower risk of voice problems. Previously, in a case-control study, Lira Luce et al selected a sample of 157 Italian teachers that were examined through a questionnaire with clinical data, the Italian validated translation of the voice handicap index (VHI), a laryngostroboscopic exam and logopedic evaluation. ${ }^{22}$ The VHI score was higher in teachers with laryngostroboscopic disorders than in the other subgroup (18.35 \pm 13.8 versus $13.45 \pm 11.46$; MannWhitney test, $p=0.026)$. Although these authors used a different questionnaire, they confirmed that the VHI score anticipates the results obtained by confirmation laryngostroboscopy. We preferred the VoiSS related to VTDS because it is psychometrically considered the most robust and extensively validated self reported voice measure available. ${ }^{23}$ Furthermore, in a sample of polish teachers, Niebudek-Bogusz et $\mathrm{al}^{24}$ evidenced a strong positive correlation between the scores of the VTDS and the total scores and the scores of physical domain of the VHI, and a moderately
Table 4 Comparison of the Mean, Number, Frequency, and Intensity of Vocal Tract Discomfort Symptoms in teachers with high risk (HRVD) and low risk (LRVD) of vocal disorders according to the emotional Score of VoiSS

\begin{tabular}{|l|l|l|l|l|l|}
\hline & \multicolumn{4}{l}{ VOISS-E } & \\
\hline & \multicolumn{4}{ll}{ HRVD } & \multicolumn{2}{l|}{ LRVD } & \\
\hline Variables & $\mathrm{M}$ & $\mathrm{SD}$ & $\mathrm{M}$ & $\mathrm{SD}$ & p-value \\
\hline Number of VTDS & 3.01 & 1.36 & 1.15 & 1.08 & $<0.001$ \\
\hline Fr. Burning & 3.03 & 1.25 & 1.73 & 1.48 & $<0.001$ \\
\hline Fr. Tightness & 2.41 & 1.48 & 0.84 & 1.08 & $<0.001$ \\
\hline Fr. Dryness & 3.05 & 1.41 & 1.38 & 1.35 & $<0.001$ \\
\hline Fr. Aching & 2.67 & 1.22 & 1.17 & 1.05 & $<0.001$ \\
\hline Fr. tickling & 2.77 & 1.81 & 1.14 & 1.28 & $<0.001$ \\
\hline Fr. Soreness & 3.41 & 1.47 & 1.19 & 1.20 & $<0.001$ \\
\hline Fr. Irritability & 3.10 & 1.59 & 1.23 & 1.19 & $<0.001$ \\
\hline $\begin{array}{l}\text { Fr. lump in } \\
\text { the throat }\end{array}$ & 2.02 & 1.70 & 0.47 & 1.03 & $<0.001$ \\
\hline Int. Burning & 3.07 & 1.23 & 1.64 & 1.34 & $<0.001$ \\
\hline Int. Tightness & 2.56 & 1.15 & 0.82 & 1.03 & $<0.001$ \\
\hline Int. Dryness & 3.05 & 1.52 & 1.33 & 1.25 & $<0.001$ \\
\hline Int. Aching & 2.77 & 1.21 & 1.31 & 1.19 & $<0.001$ \\
\hline Int. Tickling & 2.75 & 1.90 & 1.10 & 1.33 & $<0.001$ \\
\hline Int. Soreness & 3.12 & 1.42 & 1.05 & 0.98 & $<0.001$ \\
\hline Int. Irritability & 2.86 & 1.61 & 1.21 & 1.14 & $<0.001$ \\
\hline $\begin{array}{l}\text { Int. Iump in } \\
\text { the throat }\end{array}$ & 2.05 & 1.77 & 0.38 & 0.81 & $<0.001$ \\
\hline
\end{tabular}

Abbreviations: E, emotional; Fr., frequency; HRVD, high risk of vocal disorders; Int., intensity; LRVD, low risk of vocal disorders; SD, standard deviation; VoiSS, Voice Symptom Scale; VTDS, Vocal Tract Discomfort Scale.

positive correlation between the scores of VTDS and scores of the functional and emotional domains of the VHI. Although the VHI, like the VoiSS, incorporates the psychosocial impact of a dysphonia, ${ }^{23}$ only the VoiSS includes physical symptoms and shares therefore a major specificity with the VTDS in examining the frequency of voice symptoms. ${ }^{7}$ Moreover, our sample showed a moderate positive correlation between the VTDS and the total score of the VoiSS, whereas it showed a weak correlation if we considered the E-VoiSS. These data may indicate that the self-perception of the voice disorder in different aspects of the emotional life of the patients is changeable. In fact, several studies showed that the emotional impact of a voice disorder is particularly influenced by each cultural and psychosocial state, ${ }^{25}$ not correlated with the presence of physical symptoms. Rather, not always negative feelings derive from vocal disorders but, on the contrary, in a bidirectional relationship, emotional features may cause secondary dysphonia. ${ }^{26}$ Thus, from the previously published findings and those of our study, it is clear that vocal tract discomfort symptoms can impact emotions, although there is not a strong linear relationship 
Table 5 Comparison of the Mean, Number, Frequency, and Intensity of Vocal Tract Discomfort Symptoms in teachers with high risk (HRVD) and low risk (LRVD) of vocal disorders according to the physical Score of VoiSS

\begin{tabular}{|c|c|c|c|c|c|}
\hline \multirow[b]{3}{*}{ Variables } & \multicolumn{4}{|c|}{ VOISS-P } & \multirow[b]{3}{*}{ p-value } \\
\hline & \multicolumn{2}{|c|}{ HRVD } & \multicolumn{2}{|l|}{ LRVD } & \\
\hline & $\mathrm{M}$ & SD & M & SD & \\
\hline Number of VTDS & 2.97 & 1.36 & 1.35 & 1.01 & $<0.001$ \\
\hline Fr. burning & 3.17 & 1.25 & 1.77 & 1.49 & $<0.001$ \\
\hline Fr. tightness & 2.38 & 1.45 & 0.86 & 1.08 & $<0.001$ \\
\hline Fr. dryness & 2.92 & 1.30 & 1.39 & 1.34 & $<0.001$ \\
\hline Fr. aching & 2.66 & 1.28 & 1.20 & 1.07 & $<0.001$ \\
\hline Fr. tickling & 2.72 & 1.85 & 1.20 & 1.37 & $<0.001$ \\
\hline Fr. soreness & 3.24 & 1.51 & 1.25 & 1.29 & $<0.001$ \\
\hline Fr. irritability & 2.91 & 1.53 & 1.22 & 1.18 & $<0.001$ \\
\hline $\begin{array}{l}\text { Fr. lump in } \\
\text { the throat }\end{array}$ & 1.93 & 1.76 & 0.48 & 1.03 & $<0.001$ \\
\hline Int. burning & 3.14 & 1.27 & 1.68 & 1.36 & $<0.001$ \\
\hline Int. tightness & 2.66 & 1.13 & 0.84 & 1.03 & $<0.001$ \\
\hline Int. dryness & 2.92 & 1.38 & 1.34 & 1.25 & $<0.001$ \\
\hline Int. aching & 2.79 & 1.36 & 1.34 & 1.20 & $<0.001$ \\
\hline Int. tickling & 2.66 & 1.88 & 1.17 & 1.41 & $<0.001$ \\
\hline Int. soreness & 3.02 & 1.43 & 1.10 & 1.05 & $<0.001$ \\
\hline Int. irritability & 2.76 & 1.63 & 1.20 & 1.13 & $<0.001$ \\
\hline $\begin{array}{l}\text { Int. lump in } \\
\text { the throat }\end{array}$ & 1.97 & 1.76 & 0.39 & 0.81 & $<0.001$ \\
\hline
\end{tabular}

Abbreviations: Fr., frequency; HRVD, high risk of vocal disorders; Int., intensity; LRVD, low risk of vocal disorders; P, Physical; SD, standard deviation; VoiSS, Voice Symptom Scale; VTDS, Vocal Tract Discomfort Scale.

between these variables according to correlation testing. Our study evidenced like as limitation domain of VoiSS are moderately correlated with all symptoms of VTDS (weakly with aching intensity). Another study evidenced the same results and explained these results indicating that the presence of vocal tract discomfort symptoms limits the functionality of voice use in daily communication. In fact, these symptoms are associated with vocal hyperfunction, musculoskeletal strain in the neck with a higher larynx position, increasing the longitudinal tension in the vocal folds and reducing the hypopharyngeal space. ${ }^{27}$ All that behave more effort for vocal production, and, consequently, can cause limitations in the use of the voice. Another objective of our study was to hypothesize a possible correlation of VTDS and VoiSS in screening between high risk and low risk of voice disorder in teachers. As the clinical practice showed that many patients consult a specialist (doctor or speech pathologist) when their symptoms have a physical, social, emotional, or professional impact, we consider the VTDS a good screening questionnaire. The main feature/characteristic of the VTDS is that it underlines vocal tract symptoms more than VoiSS. Other authors confirm that these symptoms are important for determining the presence of a voice disorder or for the identification of a population that is at risk. ${ }^{28}$ The same auditory-perceptual evaluation, acoustic analysis, aerodynamics evaluation, and endoscopic larynx examination did not detect these pharyngeal disorders in dysphonic patients and, therefore, the VTDS showed an advantage in this sense. Thus, the VTDS appears to provide important information that complements other instruments in the context of vocal evaluations. Obviously, the questionnaires represent screening tools to identify risk categories and, therefore, require a subsequent diagnostic confirmation through phoniatric examination.

\section{Conclusion}

The current Italian law does not include any guidance regarding voice education, prevention of voice disorders and screening in subjects with high vocal loading, such as teachers. Our study showed a weak to moderate positive correlation between the VTDS and the VoiSS results. Patients with a high risk of voice disorders detected by the cutoff values of the VoiSS have VTDS scores indicating a higher number, and greater frequency and intensity of vocal tract discomfort symptoms than teachers with LRVD. These results suggest that both questionnaires could be useful for a preventive voice program for the Italian teachers for the early identification of risk categories for voice disorder.

Conflict of Interests

The authors have no conflict of interests to declare.

\section{References}

1 Amaral AC, Zambon F, Moreti F, Behlau M. Vocal tract discomfort in teachers after teaching activity. CoDAS 2017;29(02):e20160045

2 Rosen CA, Murry T. Nomenclature of voice disorders and vocal pathology. Otolaryngol Clin North Am 2000;33(05):1035-1046

3 Gama AC, Santos JN, Pedra EdeF, Rabelo AT, Magalhães MdeC, Casas EB. Vocal dose in teachers: correlation with dysphonia. CoDAS 2016;28(02):190-192

4 Roy N, Merrill RM, Thibeault S, Parsa RA, Gray SD, Smith EM. Prevalence of voice disorders in teachers and the general population. J Speech Lang Hear Res 2004;47(02):281-293

5 Cantor Cutiva LC, Vogel I, Burdorf A. Voice disorders in teachers and their associations with work-related factors: a systematic review. JCommun Disord 2013;46(02):143-155

6 Martins RH, do Amaral HA, Tavares EL, Martins MG, Gonçalves TM, Dias NH. Voice Disorders: Etiology and Diagnosis. JVoice 2016;30(06):761.e1-761.e9. Doi: 10.1016/j.jvoice.2015.09.017

7 Lopes LW, de Oliveira Florencio V, Silva POC, da Nóbrega E, Ugulino AC, Almeida AA. Vocal Tract Discomfort Scale (VTDS) and Voice Symptom Scale (VoiSS) in the Evaluation of Patients With Voice Disorders. JVoice 2019;33(03):381.e23-381.e32

8 Mozzanica F, Robotti C, Ginocchio D, et al. Cross-Cultural Adaptation and Validation of the Italian Version of the Voice Symptom Scale (IVoiSS). JVoice 2017;31(06):773.e1-773.e10. Doi: 10.1016/j. jvoice.2017.02.001

9 Woźnicka E, Niebudek-Bogusz E, Kwiecień J, Wiktorowicz J, Sliwińska-Kowalska M. Applicability of the vocal tract discomfort (VTD) scale in evaluating the effects of voice therapy of occupational voice disorders. Med Pr 2012;63(02):141-152

10 Mendes AL, Lucena BT, De Araújo AM, Melo LP, Lopes LW, Silva MF. Teacher's voice: vocal tract discomfort symptoms, vocal intensity and noise in the classroom. CoDAS 2016;28(02):168-175 
11 Naro A, Leo A, Bruno R, et al. Reducing the rate of misdiagnosis in patients with chronic disorders of consciousness: Is there a place for audiovisual stimulation? Restor Neurol Neurosci 2017;35(05): 511-526

12 Marseglia L, D’Angelo G, Impellizzeri P, et al. Neonatal stridor and laryngeal cyst: Which comes first? Pediatr Int (Roma) 2017;59 (01):115-117

13 Freni F, Galletti B, Galletti F, Dionigi G. Improved outcomes for papillary thyroid microcarcinoma care: active surveillance and case volume. Ther Adv Endocrinol Metab 2018;9(07):185-186

14 Galletti B, Bruno R, Catalano N, Cammaroto G, Freni F. Follicular carcinoma on a radio-treated ectopic lingual thyroid. Chirurgia (Turin) 2016;29(03):88-91

15 Robotti C, Mozzanica F, Pozzali I, et al. Cross-cultural Adaptation and Validation of the Italian Version of the Vocal Tract Discomfort Scale (I-VTD). J Voice 2019;33(01):115-123

16 Mathieson L. Greene and Mathieson's The Voice and Its Disorders. London and Philadelphia: Wurr Publishers Ltd; 2001 Sixth ed.

17 Mathieson L, Hirani SP, Epstein R, Baken RJ, Wood G, Rubin JS. Laryngeal manual therapy: a preliminary study to examine its treatment effects in the management of muscle tension dysphonia. J Voice 2009;23(03):353-366

18 Rodrigues G, Zambon F, Mathieson L, Behlau M. Vocal tract discomfort in teachers: its relationship to self-reported voice disorders. JVoice 2013;27(04):473-480

19 Deary IJ, Wilson JA, Carding PN, MacKenzie K. VoiSS: a patientderived voice symptom scale. J Psychosom Res 2003;54(05):483-489

20 Moreti F, Zambon F, Oliveira G, Behlau M. Cross-cultural adaptation, validation, and cutoff values of the Brazilian version of the Voice Symptom Scale-VoiSS. J Voice 2014;28(04):458-468
21 Bartz AE. Basic Statistical Concepts. 4th ed. Indianapolis: Macmillan Publishing; 1998:487

22 Lira Luce F, Teggi R, Ramella B, et al. Voice disorders in primary school teachers. Acta Otorhinolaryngol Ital 2014;34(06):412-418

23 Wilson JA, Webb A, Carding PN, Steen IN, MacKenzie K, Deary IJ. The Voice Symptom Scale (VoiSS) and the Vocal Handicap Index (VHI): a comparison of structure and content. Clin Otolaryngol Allied Sci 2004;29(02):169-174

24 Niebudek-Bogusz E, Woźnicka E, Wiktorowicz J, ŚliwińskaKowalska M. Applicability of the Polish Vocal Tract Discomfort (VTD) scale in the diagnostics of occupational dysphonia. Logoped Phoniatr Vocol 2012;37(04):151-157

25 Putnoki DS, Hara F, Oliveira G, Behlau M. Voice-related quality of life: the impact of a dysphonia according to gender, age and occupational use of voice. Rev Soc Bras Fonoaudiol 2010; 15:485-490

26 Almeida LNA, Lopes LW, Costa DB, Silva EG, da Cunha GMS, De Almeida AAF. Vocal and emotional features of teachers and nonteachers with low and high anxiety. Audiol Commun Res 2014; 19:179-185

27 Guzman M, Castro C, Testart A, Muñoz D, Gerhard J. Laryngeal and pharyngeal activity during semioccluded vocal tract postures in subjects diagnosed with hyperfunctional dysphonia. J Voice 2013; 27(06):709-716

28 Pernambuco LdeA, Espelt A, Magalhães Júnior HV, Cavalcanti RV, de Lima KC. Screening for Voice Disorders in Older Adults (Rastreamento de Alterações Vocais em Idosos-RAVI)-Part I: Validity Evidence Based on Test Content and Response Processes. JVoice 2016;30(02):246.e9-246.e17. Doi: 10.1016/j.jvoice. 2015.04.008 